\title{
Infinite hierarchy of nonlinear Schrödinger equations and their solutions
}

\author{
A. Ankiewicz, ${ }^{1}$ D. J. Kedziora, ${ }^{1}$ A. Chowdury, ${ }^{1}$ U. Bandelow, ${ }^{2}$ and N. Akhmediev ${ }^{1}$ \\ ${ }^{1}$ Optical Sciences Group, Research School of Physics and Engineering, The Australian National University, Canberra, \\ Australian Capital Territory 0200, Australia \\ ${ }^{2}$ Weierstrass Institute for Applied Analysis and Stochastics, Mohrenstrasse 39, 10117 Berlin, Germany
}

(Received 26 September 2015; published 11 January 2016)

\begin{abstract}
We study the infinite integrable nonlinear Schrödinger equation hierarchy beyond the Lakshmanan-PorsezianDaniel equation which is a particular (fourth-order) case of the hierarchy. In particular, we present the generalized Lax pair and generalized soliton solutions, plane wave solutions, Akhmediev breathers, Kuznetsov-Ma breathers, periodic solutions, and rogue wave solutions for this infinite-order hierarchy. We find that "even- order" equations in the set affect phase and "stretching factors" in the solutions, while "odd-order" equations affect the velocities. Hence odd-order equation solutions can be real functions, while even-order equation solutions are always complex.
\end{abstract}

DOI: 10.1103/PhysRevE.93.012206

\section{INTRODUCTION}

It is well-known that the one-dimensional fundamental nonlinear Schrödinger equation (NLSE) is integrable [1]. This fact has allowed the achievement of significant progress in the analysis of nonlinear optics, water waves, Bose-Einstein condensates, and many other fields of nonlinear physics. The possibility of writing solutions of the NLSE in an analytical form has stimulated numerous experimental works in these areas. Initial developments in soliton solutions have been strengthened recently by the advances in breather solutions. Various families of solutions are presented in Ref. [2].

Although the NLSE is one of the fundamental equations in physics, it is not the only one which is integrable. In particular, various extensions of the NLSE are known. For example, Painlevé analysis of deformed NLS and Hirota equations are given in Ref. [3]. Kano [4] considered small perturbations of the NLSE that allowed him to keep the modified equation nearly integrable. Such extensions expand the areas of applicability of integrable equations and provide efficient ways to apply evolution equations in practice. For example, they may help to clarify the physics of wave blow-up and collapse phenomena [5], as higher intensities require higher-order terms to be included.

In the present work, we provide an extension of the NLSE to infinite-order equations that comprise the NLSE hierarchy. Namely, we consider extensions of the NLSE where additional terms can have arbitrarily large coefficients. This extension creates the infinite hierarchy of equations that are integrable with an infinite number of arbitrary real coefficients. The additional terms in the equation include higher-order dispersion of all orders and higher-order dispersion of nonlinear terms. The arbitrariness of coefficients allows us to go well beyond the simple NLSE. We define the invariant integrands of the NLSE as

$$
p_{j+1}=\psi \frac{\partial}{\partial t}\left(\frac{p_{j}}{\psi}\right)+\sum_{j_{1}+j_{2}=j}\left(p_{j_{1}} p_{j_{2}}\right),
$$

where $j=1,2,3, \cdots, \infty$ and $j_{1}$ and $j_{2}$ are nonzero positive integers which add up to $j$, noting that order is important. For example, if $j=1$ there are no such integers and so the right summation is zero, while for $j=4$, we have $\left(j_{1}, j_{2}\right)=$
$(1,3),(3,1)$, and $(2,2)$, so that

$$
\sum_{j_{1}+j_{2}=4}\left(p_{j_{1}} p_{j_{2}}\right)=2 p_{1} p_{3}+p_{2}^{2} .
$$

We take $p_{1}=|\psi|^{2}$ to start with. Hence, the first few functionals are

$$
\begin{aligned}
& p_{2}=\psi \psi_{t}^{*}, \\
& p_{3}=|\psi|^{4}+\psi \psi_{t t}^{*}, \\
& p_{4}=\psi\left[\psi_{t}\left(\psi^{*}\right)^{2}+4 \psi_{t}^{*}|\psi|^{2}+\psi_{t t t}^{*}\right] .
\end{aligned}
$$

With this formulation, all signs are positive. Now, we define the $j$ th operator in the NLS hierarchy as

$$
K_{j}\left(\psi, \psi^{*}\right)=(-1)^{j} \frac{\delta}{\delta \psi^{*}}\left[\int p_{j+1} d t\right],
$$

where we have taken the functional derivative of the invariant to get the higher-order operator. Again, all signs are positive in each $K_{j}$. For example, $K_{2}=\psi_{t t}+2 \psi|\psi|^{2}$, which is easily recognizable as an NLSE operator.

For higher orders, the $j$ th operator $(j \geqslant 3)$ can be presented in the following form:

$$
\begin{aligned}
K_{j}= & \frac{\partial^{j} \psi}{\partial t^{j}}+2 j|\psi|^{2} \frac{\partial^{j-2} \psi}{\partial t^{j-2}}+\left[1+(-1)^{j}\right] \psi^{2} \frac{\partial^{j-2} \psi^{*}}{\partial t^{j-2}} \\
& +j(j-3) \psi \psi_{t}^{*} \frac{\partial^{j-3} \psi}{\partial t^{j-3}}+\cdots .
\end{aligned}
$$

There are only two terms when $j=3$, as the last two terms reduce to zero: $K_{3}=\psi_{t t t}+6|\psi|^{2} \psi_{t}$. For $j \geqslant 4$, the next term to be added is

$$
2\left[j-1-(-1)^{j}\right] \psi \psi_{t} \frac{\partial^{j-3} \psi^{*}}{\partial t^{j-3}} .
$$

For $j \geqslant 5$, the next term to be added is

$$
j(j-1) \psi_{t} \psi^{*} \frac{\partial^{j-3} \psi}{\partial t^{j-3}} .
$$

Finally, the term with no derivative in $K_{j}$ is

$$
\frac{j !}{[(j / 2) !]^{2}} \psi|\psi|^{j}
$$

if $j$ is even, $j=2,4,6, \cdots$, and zero if $j$ is odd. 
The equation which includes the whole infinite hierarchy is

$$
F[\psi(x, t)]=i \psi_{x}+\sum_{j=1}^{\infty}\left(\alpha_{2 j} K_{2 j}-i \alpha_{2 j+1} K_{2 j+1}\right)=0,
$$

where each coefficient $\alpha_{j}, j=2,3,4,5, \cdots, \infty$, is an arbitrary real number. In all expressions here, $x$ is the propagation variable and $t$ is the transverse variable (time in a moving frame), with the function $|\psi(x, t)|$ being the envelope of the waves.

In Ref. [2] and many papers, including Refs. [6-8], we have taken $\alpha_{2}=\frac{1}{2}$. This normalization has certain convenient features. For example, rogue wave triplets with this scaling are circular rather than elliptical in the $(x, t)$ plane [9]. On the other hand, some authors, e.g., Zakharov and Shabat [1] and Kano [4], set $\alpha_{2}=1$. Any value of $\alpha_{2}$ can be used in our present work [10-13], including zero. Hence, our solutions cover equations like $\psi_{x}-\alpha_{3}\left(\psi_{t t t}+6|\psi|^{2} \psi_{t}\right)=0$, which do not involve the basic NLSE operator at all. The latter is a significant advance over previous works.

Thus, the whole equation takes the following form:

$$
\begin{aligned}
F[\psi(x, t)]= & i \psi_{x}+\alpha_{2} K_{2}[\psi(x, t)]-i \alpha_{3} K_{3}[\psi(x, t)] \\
& +\alpha_{4} K_{4}[\psi(x, t)]-i \alpha_{5} K_{5}[\psi(x, t)] \\
& +\alpha_{6} K_{6}[\psi(x, t)]-i \alpha_{7} K_{7}[\psi(x, t)] \\
& +\alpha_{8} K_{8}[\psi(x, t)]-i \alpha_{9} K_{9}[\psi(x, t)] \\
& +\cdots=0
\end{aligned}
$$

where the combined operator $F[\psi(x, t)]$ represents the whole hierarchy of integrable equations.

In the lowest, second order, we obtain the fundamental nonlinear Schrödinger equation:

$$
i \psi_{x}+\alpha_{2} K_{2}=i \psi_{x}+\alpha_{2}\left(\psi_{t t}+2 \psi|\psi|^{2}\right)=0 .
$$

Keeping additionally the third-order operator $K_{3}$, we obtain the Hirota equation:

$$
i \psi_{x}+\alpha_{2}\left(\psi_{t t}+2 \psi|\psi|^{2}\right)-i \alpha_{3}\left[\psi_{t t t}+6|\psi|^{2} \psi_{t}\right]=0 .
$$

In the next generalization, we keep $K_{4}$ as the fourth-order $(j=$ 4) operator. It is known as the Lakshmanan-Porsezian-Daniel (LPD) operator (starting with the fourth-order derivative):

$$
\begin{aligned}
K_{4}[\psi(x, t)]= & \psi_{t t t t}+8|\psi|^{2} \psi_{t t}+6 \psi|\psi|^{4}+4 \psi\left|\psi_{t}\right|^{2} \\
& +6 \psi_{t}^{2} \psi^{*}+2 \psi^{2} \psi_{t t}^{*} .
\end{aligned}
$$

Continuing the process, we can keep $K_{5}$ as the fifth-order $(j=5)$, i.e., quintic, operator (starting with the fifth-order derivative):

$$
\begin{aligned}
K_{5}[\psi(x, t)]= & \psi_{t t t t t}+10|\psi|^{2} \psi_{t t t}+30|\psi|^{4} \psi_{t}+10 \psi \psi_{t} \psi_{t t}^{*} \\
& +10 \psi \psi_{t}^{*} \psi_{t t}+20 \psi^{*} \psi_{t} \psi_{t t}+10 \psi_{t}^{2} \psi_{t}^{*} .
\end{aligned}
$$

This expression can be written in a shorter form:

$$
\begin{aligned}
K_{5}[\psi(x, t)]= & \psi_{t t t t t}+10|\psi|^{2} \psi_{t t t}+10\left(\psi\left|\psi_{t}\right|^{2}\right)_{t} \\
& +20 \psi^{*} \psi_{t} \psi_{t t}+30|\psi|^{4} \psi_{t} .
\end{aligned}
$$

The quintic equation has been considered, in a different context, by Hoseini and Marchant [14]. Further, $K_{6}$ is the sixthorder $(j=6)$, i.e., sextic, operator (starting with the sixthorder derivative):

$$
\begin{aligned}
K_{6}[\psi(x, t)]= & \psi_{t t t t t t}+\left[60 \psi^{*}\left|\psi_{t}\right|^{2}+50\left(\psi^{*}\right)^{2} \psi_{t t}+2 \psi_{t t t t}^{*}\right] \psi^{2} \\
& +\psi\left[12 \psi^{*} \psi_{t t t t}+8 \psi_{t} \psi_{t t t}^{*}+22\left|\psi_{t t}\right|^{2}\right] \\
& +\psi\left[18 \psi_{t t t} \psi_{t}^{*}+70\left(\psi^{*}\right)^{2} \psi_{t}^{2}\right]+20\left(\psi_{t}\right)^{2} \psi_{t t}^{*} \\
& +10 \psi_{t}\left[5 \psi_{t t} \psi_{t}^{*}+3 \psi^{*} \psi_{t t t}\right]+20 \psi^{*} \psi_{t t}^{2} \\
& +10 \psi^{3}\left[\left(\psi_{t}^{*}\right)^{2}+2 \psi^{*} \psi_{t t}^{*}\right]+20 \psi|\psi|^{6} .
\end{aligned}
$$

We present the heptic and octic operators in the Appendix.

We repeat, the coefficients $\alpha_{j}$ are arbitrary real constants. They do not have to be small. This allows us to go well beyond the simple extension of the NLSE with corrective and perturbative terms. In the particular case when only $\alpha_{3}$ is nonzero, the equation is known as the Hirota equation $[6,15]$. Furthermore, when only $\alpha_{4}$ is nonzero, the equation is known as the LPD equation [16-18]. In this case, the coefficients within the $K_{4}$ operator (8) were found using Painlevé analysis of the equation describing the Heisenberg spin chain. Thus, particular cases in the hierarchy have physical relevance. The equation when two coefficients $\alpha_{3}$ and $\alpha_{4}$ are arbitrary has been considered earlier in Refs. [7,8]. In particular, soliton solutions of this equation are given in Ref. [7], while rogue wave solutions are presented in Ref. [8]. In those papers, $\alpha_{4}$ is denoted by $\gamma$. The $\mathrm{KdV}$ is studied in $[19,20]$.

We believe that the sextic, heptic, and octic operators of the NLS hierarchy are presented here for the first time. Although we do not present here the ninth-order operator $K_{9}[\psi]$, with coefficient $\alpha_{9}$ to save space, the results we give for first-order solitons and rogue waves does include it and all higher orders to infinity.

\section{GENERAL OBSERVATIONS}

\section{A. Scaling}

If we have a solution, $\psi\left(x, t ; \alpha_{2}, \alpha_{3}, \alpha_{4}, \ldots\right)$, of the full equation, then we can generate a scaled solution by multiplying the function by an arbitrary real constant, $c$, multiplying $t$ by $c$, leaving $x$ unchanged, and multiplying each $\alpha_{j}$ in the solution by $c^{j}$. Hence the new solution is $c \psi\left(x, c t ; c^{2} \alpha_{2}, c^{3} \alpha_{3}, c^{4} \alpha_{4}, \ldots\right)$. If all $\alpha_{j}=0$ for $j \geqslant 3$, i.e., we have the fundamental NLSE only, then the scaling $\alpha_{2} \rightarrow$ $c^{2} \alpha_{2}$ is equivalent to scaling $x$ by a factor of $c^{2}$, thus agreeing with the well-known scaling of NLSE solutions (e.g., see [21] and Eq. (2.3) of Ref. [2]). However, when more operators are included in the equation, it is important to note that the $\alpha_{j}$ 's in the solution are scaled, not the variable $x$. This will be clear from the solutions analyzed in this paper. This scaling is not trivial, and so we retain the $c$ factors throughout the solutions, for ease of use.

\section{B. Odd-numbered equations}

First, we make some general observations. If all evenlabeled coefficients are zero, i.e. $\alpha_{2 n}=0, n=1,2,3, \ldots, \infty$, 
then we have

$$
\psi_{x}=\sum_{j=1}^{\infty} \alpha_{2 j+1} K_{2 j+1}
$$

These can have real-valued solutions. For example, the first such equation is $\psi_{x}=\alpha_{3}\left(\psi_{t t t}+6|\psi|^{2} \psi_{t}\right)$. If we assume that $\psi=f(y)$ is a real even function where $y=t+x v_{3}$, then for a localized solution $\left[f(y) \rightarrow 0\right.$ for $y \rightarrow \infty$ ], we have $v_{3} f=$ $\alpha_{3}\left(f^{\prime \prime}+2 f^{3}\right)$. For convenience, we set $f(0)=1$. This shows that $v_{3}=\alpha_{3}$ and $f^{\prime}(y)=f \sqrt{1-f^{2}}$. Hence $f=\operatorname{sech}(y)=$ $\operatorname{sech}\left(t+x v_{3}\right)$.

Similarly, the $j=2$ equation, with $y=t+x v_{5}$ reduces to $v_{5} f^{\prime}=\alpha_{5}\left(f_{5 y}+10 f^{2} f_{3 y}+30 f^{4} f^{\prime}+40 f f^{\prime} f_{2 y}+\right.$ $\left.10 f_{y}^{3}\right)$. Then $v_{5}=\alpha_{5}$ and $f=\operatorname{sech}\left(t+x v_{5}\right)$. This pattern will be seen later with more complicated solutions. We will be able to plot $\psi$ rather than just $|\psi|$ for these solutions.

\section{Even-numbered equations}

If all odd-labeled coefficients are zero, i.e., $\alpha_{2 n+1}=0, n=$ $1,2,3, \ldots, \infty$ then the equation becomes

$$
i \psi_{x}+\sum_{j=1}^{\infty} \alpha_{2 j} K_{2 j}=0
$$

Now the solutions take the form $\psi=e^{i \phi x} g(t)$. In the NLSE case, when $j=1$, for the localized solution which is even in $t$, we have $\alpha_{2}\left(g_{t}^{2}+g^{4}\right)=\phi g$. For convenience, we now take $g(0)=1$. This shows that $\phi=\alpha_{2}$ and $g^{\prime}(t)=g \sqrt{1-g^{2}}$. Hence $g=\operatorname{sech}(t)$ and $\psi=e^{i \alpha_{2} x} \operatorname{sech}(t)$.

Similarly, for the $j=2$ equation, we have $\alpha_{4}\left(g_{4 t}+\right.$ $\left.8 g^{2} g_{2 t}+6 g^{5}+10 g g_{t}^{2}+2 g^{2} g_{2 t}\right)=\phi g$ and solution $\psi=$ $e^{i \alpha_{4} x} \operatorname{sech}(t)$. Again, this structure will be seen for other types of solutions.

\section{Plane wave solutions}

In order to illustrate the usefulness of the approach, we start with the simplest plane wave solution of the extended NLS equation. If the solution $\psi$ is independent of $t$, then we see from Eq. (5) that

$$
i \psi_{x}+\psi \sum_{n=1}^{\infty}\left(\begin{array}{c}
2 n \\
n
\end{array}\right) \alpha_{2 n}|\psi|^{2 n}=0,
$$

where $\left(\begin{array}{c}2 n \\ n\end{array}\right)$ is a binomial coefficient. So

$$
i \psi_{x}+2 \alpha_{2} \psi|\psi|^{2}+6 \alpha_{4} \psi|\psi|^{4}+20 \alpha_{6} \psi|\psi|^{6}+\cdots=0 .
$$

Thus, for the unit-background forward-propagating plane wave solution to Eq. (7), $\psi_{p}=\exp (i \phi x)$, we have (with $j=2 n)$

$\phi=2 \alpha_{2}+6 \alpha_{4}+20 \alpha_{6}+\cdots=\sum_{n=1}^{\infty}\left(\begin{array}{c}2 n \\ n\end{array}\right) \alpha_{2 n}=\sum_{n=1}^{\infty} \frac{(2 n) !}{(n !)^{2}} \alpha_{2 n}$.
Thus, for an arbitrary background of the plane wave, we can write the solution as

$$
\begin{aligned}
\psi_{p}= & c \exp \left(i x c^{2} \sum_{n=1}^{\infty} \frac{(2 n) !}{(n !)^{2}} \alpha_{2 n} c^{2 n-2}\right) \\
= & c \exp \left[i x c ^ { 2 } \left(2 \alpha_{2}+6 c^{2} \alpha_{4}+20 c^{4} \alpha_{6}\right.\right. \\
& \left.\left.+70 c^{6} \alpha_{8}+252 c^{8} \alpha_{10}+\cdots\right)\right],
\end{aligned}
$$

recalling that $\alpha_{2}$ does not have to be $1 / 2$.

Here $c$ is the arbitrary amplitude of the plane wave and the series in Eq. (4) contains even coefficients of Eq. (7). The simple nature of the scaling is apparent, with arbitrary background level $c$ causing each coefficient $\alpha_{2 n}$ to be multiplied by $c^{2 n}$. The expression (4) represents the solution of Eq. (7) of any order up to infinite one. The presence of only even terms in this expression is related to the fact that we deal with the forward-propagating wave. Any skewness in the $(x, t)$ plane would result in the addition of odd terms. We do not present this case as this would go beyond the simplicity of our illustrative example. In order to construct more complicated solutions of Eq. (7), we have to find its Lax pair. These solutions can also be found for the equation with an infinite number of terms.

\section{E. First-order soliton solutions}

The first-order soliton of Eq. (7), taking $\alpha_{2}$ and all other coefficients $\alpha_{j}$ to be arbitrary, is

$$
\psi_{s}=c \exp \left(i x \phi_{s}\right) \operatorname{sech}\left(c t+x v_{s}\right),
$$

where the phase is

$$
\begin{aligned}
\phi_{s} & =\sum_{n=1}^{\infty} \alpha_{2 n} c^{2 n} \\
& =c^{2}\left(\alpha_{2}+c^{2} \alpha_{4}+c^{4} \alpha_{6}+c^{6} \alpha_{8}+c^{8} \alpha_{10}+\cdots\right),
\end{aligned}
$$

and where the velocity is

$$
\begin{aligned}
v_{s} & =\sum_{n=1}^{\infty} \alpha_{2 n+1} c^{2 n+1} \\
& =c^{3}\left(\alpha_{3}+c^{2} \alpha_{5}+c^{4} \alpha_{7}+c^{6} \alpha_{9}+\cdots\right) .
\end{aligned}
$$

The background level $c$ is arbitrary. It is clear from the expression that the velocity depends on the third-, fifth-, seventh-, and ninth-order coefficients $\alpha_{2 n+1}$, while the phase depends on the fourth-, sixth-, and eighth-order coefficients $\alpha_{2 n}$. Plainly, for the unit background, each term has a unit coefficient. When only $\alpha_{3}$ and $\alpha_{4}$ are nonzero, it reduces to a result in Ref. [7]. So this solution applies for infinitely many orders in the original equation. It confirms and generalizes the brief derivations on odd- and even-numbered equations above.

\section{GENERALIZED ROGUE WAVES AND RELATED SOLUTIONS}

Again, we allow for all operator coefficients $\left(\alpha_{j}, j=\right.$ $3,4,5, \cdots, \infty)$ to be arbitary. Then

$$
\psi(x, t)=c\left[4 \frac{1+2 i B_{r} x}{D(x, t)}-1\right] e^{i \phi_{r} x},
$$




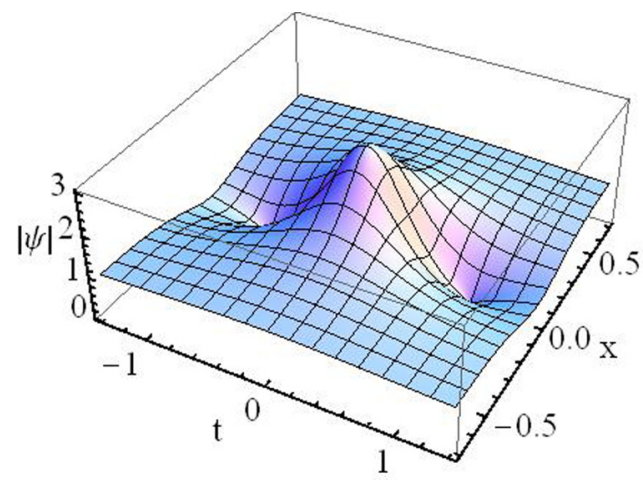

FIG. 1. Plot of the rogue wave, Eq .(15), solution of Eq. (7), with $c=1, \alpha_{4}=\frac{1}{4}$, and all other $\alpha_{j}$ 's being zero.

where $D(x, t)=1+4 B_{r}^{2} x^{2}+4\left(c t+v_{r} x\right)^{2}$ and

$$
\begin{aligned}
B_{r}= & \sum_{n=1}^{\infty} \frac{n(2 n) !}{(n !)^{2}} \alpha_{2 n} c^{2 n} \\
= & 2 c^{2}\left(\alpha_{2}+6 c^{2} \alpha_{4}+30 c^{4} \alpha_{6}+140 c^{6} \alpha_{8}\right. \\
& \left.+630 c^{8} \alpha_{10}+\cdots\right) .
\end{aligned}
$$

Here $c$ is the arbitrary background level. The coefficient $\phi$ in the exponential factor is then equal to

$$
\begin{aligned}
\phi_{r} & =c^{2} \sum_{n=1}^{\infty} \frac{(2 n) !}{(n !)^{2}} \alpha_{2 n} c^{2 n-2} \\
& =2 c^{2}\left(\alpha_{2}+3 c^{2} \alpha_{4}+10 c^{4} \alpha_{6}+35 c^{6} \alpha_{8}+126 c^{8} \alpha_{10}+\cdots\right) .
\end{aligned}
$$

Finally, the velocity is

$$
\begin{aligned}
v_{r}= & \sum_{n=1}^{\infty} \frac{(2 n+1) !}{(n !)^{2}} \alpha_{2 n+1} c^{2 n+1} \\
= & 2 c^{3}\left(3 \alpha_{3}+15 c^{2} \alpha_{5}+70 c^{4} \alpha_{7}+315 c^{6} \alpha_{9}\right. \\
& \left.+1386 c^{8} \alpha_{11}+\cdots\right) .
\end{aligned}
$$

The velocity clearly depends only on the coefficients of odd-order operators: the Hirota operator with $v=6 c^{3} \alpha_{3}$ when the other $\alpha_{j}$ 's are zero, the fifth-order operator (quintic, with $v=30 c^{5} \alpha_{5}$ when the other $\alpha_{j}$ 's are zero), the seventh-order operator (heptic, with $v=140 c^{7} \alpha_{7}$ when the other $\alpha_{j}$ 's are zero), etc. We note that the exponential factor, $\phi$, and the stretching factor, $B_{r}$, here depend only on the coefficients of even-order operators. When $\alpha_{j}=0$, for all $j>4$, it reduces to a result in Ref. [8].

If we have only even-numbered equations, then $\phi_{r}$ and $B_{r}$ are nonzero, and we obtain complex-valued, zero-velocity solutions resembling that of the NLSE (which is the $\alpha_{2}$ case). An example is given in Fig. 1.

If we have only odd-numbered equations (see Sec. II B), then $\phi_{r}=B_{r}=0$, and we obtain the real-valued solution

$$
\psi(x, t)=c\left[\frac{4}{4\left(c t+v_{r} x\right)^{2}}-1\right] .
$$

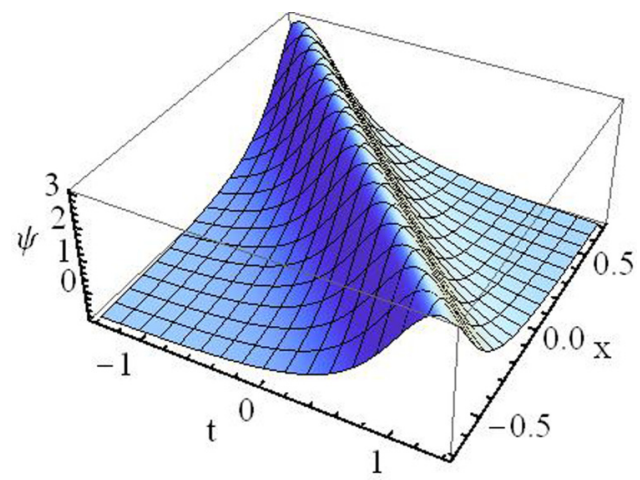

FIG. 2. Plot of the moving soliton on a background, Eq. (15), with $c=1, \alpha_{5}=\frac{1}{16}$, and all other $\alpha_{j}$ 's being zero.

Then, along the diagonal line $c t+v_{r} x=0$, we have $\psi(x, t)=$ $3 c$. So, this solution resembles a moving soliton on a background (though the shape is different from the "sech" function) and does not have the single peak which is a feature of solutions of the full equation which contains at least one even-labeled term. An example is given in Fig. 2. Earlier works, e.g., Ref. [6], included both $\alpha_{2}$ and $\alpha_{3}$ terms and hence found rogue waves with a single maximum.

If we have at least one even-numbered equation with at least one odd-numbered equation, the resulting solution looks like an NLSE rogue wave [22], with the central part having a velocity (see Ref. [6]). An example is given in Fig. 3. We stress that this is a remarkably simple result for an equation that can contain hundreds of terms, each with various derivatives. It could help explain the appearance of rogue waves in a multitude of physical, biological, financial, and social situations, going well beyond the $j=3$ and 4 cases that have been previously analyzed.

\section{GENERALIZED AKHMEDIEV BREATHERS AND RELATED SOLUTIONS}

The basic NLSE breather explains the evolution of modulation instability (e.g., see Sec. 3.7 of Ref. [2]). Here, we consider all odd- and even-order equations. The odd-order equations basically modify the breather velocity when compared to the basic NLSE breather, as is already known for the Hirota $\left(\alpha_{3} \neq 0\right)$ case. On the other hand, the even-order equations

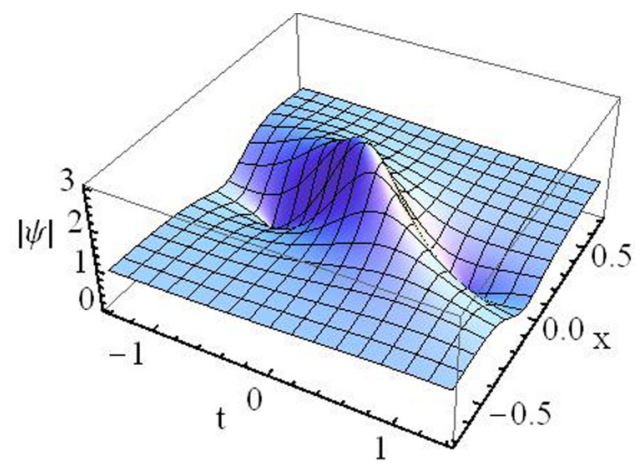

FIG. 3. Plot of the rogue wave, Eq. (15), with $c=1, \alpha_{4}=1 / 4$, $\alpha_{5}=\frac{1}{16}$, and all other $\alpha_{j}$ 's being zero. 
basically modify the phase of the basic NLSE breather and introduce a "stretching factor" in $x$ in the nonphase part of the solution. We take an arbitrary background, i.e., any real $c$, while noting that the scaling is relatively simple once the $c=1$ case is known. Thus, the general breather on the arbitrary background $c$ is

$$
\psi_{b}=c e^{i x \phi}\left(1+\frac{\kappa\left[\kappa C(x)+i \sqrt{4-\kappa^{2}} S(x)\right]}{\sqrt{4-\kappa^{2}} \cos \left[\kappa\left(c t+v_{b} x\right)\right]-2 C(x)}\right),
$$

where

$$
\begin{aligned}
& C(x)=\cosh \left(B_{b} \kappa \sqrt{1-\frac{\kappa^{2}}{4} x}\right), \\
& S(x)=\sinh \left(B_{b} \kappa \sqrt{1-\frac{\kappa^{2}}{4} x}\right),
\end{aligned}
$$

with $\kappa$ being an arbitrary real frequency in the range of modulation instability, i.e., $0<\kappa<2$.

Now the velocity is

$$
v_{b}=\sum_{n=1}^{\infty} \alpha_{2 n+1} c^{2 n+1} \frac{(2 n+1) !}{n !}\left(\sum_{r=0}^{n} \frac{(-1)^{r} \kappa^{2 r} r !}{(n-r) !(2 r+1) !}\right) .
$$

We now sum the series on the right, obtaining a closed form result:

$$
v_{b}=\sum_{n=1}^{\infty} \alpha_{2 n+1} c^{2 n+1} \frac{(2 n+1) !}{(n !)^{2}}{ }_{2} F_{1}\left(1,-n ; \frac{3}{2} ; \frac{\kappa^{2}}{4}\right),
$$

where ${ }_{2} F_{1}$ is the hypergeometric function [23]. In our range, if $\kappa$ is small, then this function can be approximated:

$$
{ }_{2} F_{1}\left(1,-n ; \frac{3}{2} ; \frac{\kappa^{2}}{4}\right) \approx 1-\frac{n}{6} \kappa^{2}+\frac{n}{60}(n-1) \kappa^{4}+\cdots \text {. }
$$

In fact, for any $\kappa$ in our range, ${ }_{2} F_{1}\left(1,-n ; \frac{3}{2} ; \frac{\kappa^{2}}{4}\right)$ is exactly a polynomial in $\kappa$ with $n+1$ terms, with the highest power being $\kappa^{2 n}$. Thus,

$$
\begin{aligned}
v_{b}= & \alpha_{3} c^{3}\left(6-\kappa^{2}\right)+\alpha_{5} c^{5}\left(30-10 \kappa^{2}+\kappa^{4}\right) \\
& +\alpha_{7} c^{7}\left(140-70 \kappa^{2}+14 \kappa^{4}-\kappa^{6}\right)+\alpha_{9} c^{9}(630 \\
& \left.-420 \kappa^{2}+126 \kappa^{4}-18 \kappa^{6}+\kappa^{8}\right)+\alpha_{11} c^{11}(2772 \\
& \left.-2310 \kappa^{2}+924 \kappa^{4}-198 \kappa^{6}+22 \kappa^{8}-\kappa^{10}\right)+\cdots
\end{aligned}
$$

For $\kappa \rightarrow 0$, from Eqs.(20) and (21), we have

$$
\begin{aligned}
v_{b}= & \sum_{n=1}^{\infty} \alpha_{2 n+1} c^{2 n+1} \frac{(2 n+1) !}{(n !)^{2}}=6 \alpha_{3} c^{3}+30 \alpha_{5} c^{5} \\
& +140 \alpha_{7} c^{7}+630 \alpha_{9} c^{9}+2772 \alpha_{11} c^{11}+\cdots
\end{aligned}
$$

agreeing with the rogue wave result, Eq. (18). Thus, the velocity given by Eq. (18) is the low-frequency $(\kappa \rightarrow 0)$ limit (for any $c$ ) of Eq. (20).

Similarly, the stretching factor is given by

$$
\begin{aligned}
B_{b} & =2 \sum_{n=0}^{\infty} \alpha_{2 n+2} c^{2 n+2} \frac{(2 n+1) !}{(n !)^{2}}{ }_{2} F_{1}\left(1,-n ; \frac{3}{2} ; \frac{\kappa^{2}}{4}\right) \\
& =2\left[\alpha_{2} c^{2}+\alpha_{4} c^{4}\left(6-\kappa^{2}\right)+\alpha_{6} c^{6}\left(30-10 \kappa^{2}+\kappa^{4}\right)\right.
\end{aligned}
$$

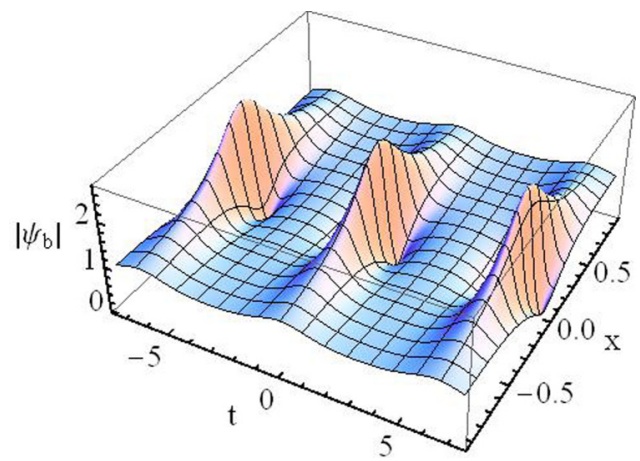

FIG. 4. Plot of the complex Akhmediev breather, Eq. (19), solution of Eq. (7), with $\kappa=1, c=1, \alpha_{4}=\frac{1}{4}$, and all other $\alpha_{j}$ 's being zero.

$$
\begin{aligned}
& +\alpha_{8} c^{8}\left(140-70 \kappa^{2}+14 \kappa^{4}-\kappa^{6}\right)+\alpha_{10} c^{10}(630 \\
& \left.-420 \kappa^{2}+126 \kappa^{4}-18 \kappa^{6}+\kappa^{8}\right)+\alpha_{12} c^{12}(2772 \\
& \left.\left.-2310 \kappa^{2}+924 \kappa^{4}-198 \kappa^{6}+22 \kappa^{8}-\kappa^{10}\right)+\cdots\right] .
\end{aligned}
$$

The $\kappa \rightarrow 0$ limit is

$$
B_{b}(\kappa=0)=2 \sum_{n=0}^{\infty} \alpha_{2 n+2} c^{2 n+2} \frac{(2 n+1) !}{(n !)^{2}},
$$

agreeing with the rogue wave result, Eq. (16). The phase is:

$$
\phi=\sum_{n=1}^{\infty} \alpha_{2 n} c^{2 n} \frac{(2 n) !}{(n !)^{2}}=2\left(\alpha_{2} c^{2}+3 \alpha_{4} c^{4}+10 \alpha_{6} c^{6}+\cdots\right)
$$

Note that the phase matches that of the plane wave solution, Eq. (13), and the rogue wave, Eq. (17). Thus, the rogue wave, Eq. (15), can also be obtained as the low-frequency $(\kappa \rightarrow 0)$ limit of the breather given by Eq. (19).

We have $|\phi(0,0)|=|c|\left(1+\sqrt{4-\kappa^{2}}\right)$; this decreases from a maximum of $3|c|$ when $\kappa=0$ to a minimum of $|c|$ when $\kappa=2$. Again, if we have only even numbered equations, then $v_{b}=0$, and the breather solution resembles that of the NLSE. An example is given in Fig. 4.

If we have only odd-numbered equations, then $\phi_{b}=B_{b}=$ 0 , and the solution $\psi_{b}(x, t)$ of Eq. (19) becomes real-valued. An example is given in Fig. 5. It does not "breathe" and so we can describe it as a solution related to a breather. If at least

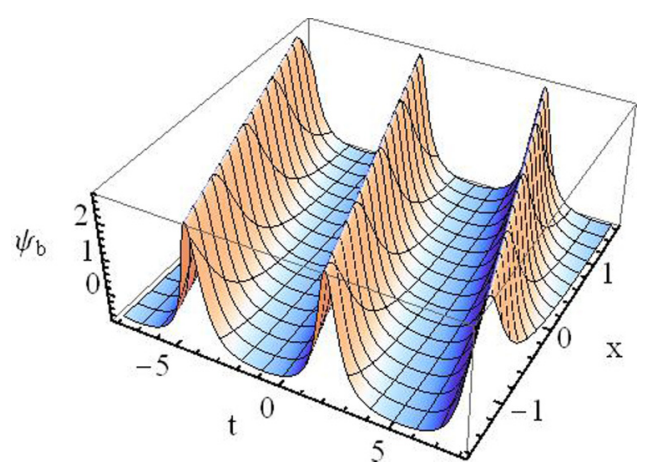

FIG. 5. Plot of the real-valued nonbreathing solution related to the Akhmediev breathers, Eq. (19). It is a solution of Eq. (7), with $\kappa=1, c=-1, \alpha_{5}=\frac{1}{16}$, and all other $\alpha_{j}$ 's being zero. 


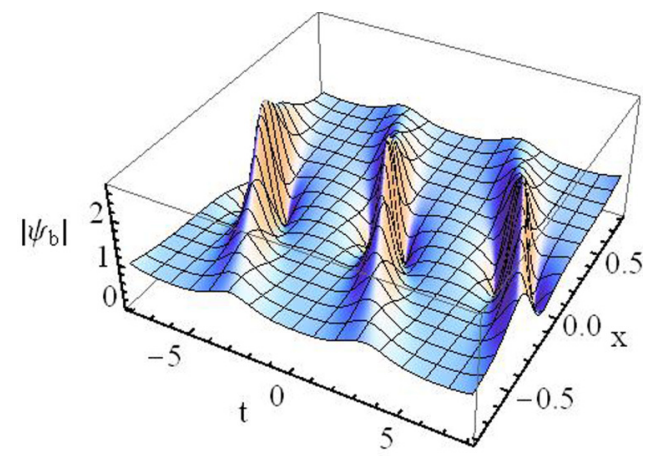

FIG. 6. Plot of the complex Akhmediev breather, Eq. (19), solution of Eq. (7), with $\kappa=1, c=1, \alpha_{4}=\frac{1}{4}, \alpha_{5}=\frac{1}{5}$, and all other $\alpha_{j}$ 's being zero. one even and one odd coefficient are nonzero, then $\phi_{b}, B_{b}$, and $v_{b}$ are all nonzero. The example of this solution is shown in Fig. 6. It is similar to the one in Fig. 4 but has nonzero velocity.

Beyond the NLSE solution, only the Hirota case [6] when $v_{b}=\alpha_{3}\left(6-\kappa^{2}\right)$ and fourth-order case [24] were previously known. This new solution can provide a considerable extension of applicability to problems of modulation instability in physics, chemistry, etc.

\section{GENERALIZED KUZNETSOV-MA BREATHERS AND MOVING SOLITONS}

The NLSE Kuznetsov-Ma breather is given by Eq. (3.63) of Ref. [2]. The generalized Kuznetsov-Ma breather can be written as

$$
\psi_{m}=c \sqrt{2} e^{i x\left(\phi_{m}\right)} \frac{2\left(1-a_{1}\right) C_{s}(x)-\sqrt{2} \sqrt{a_{1}} C_{m}(x, t)+2 i \sqrt{1-2 a_{1}} S_{m}(x)}{\sqrt{2} C_{m}(x, t)-2 \sqrt{a_{1}} C_{s}(x)},
$$

where

$$
\begin{aligned}
C_{m}(x, t) & =\cosh \left[2 \sqrt{1-2 a_{1}}\left(c t+v_{m} x\right)\right], \\
C_{s}(x) & =\cos \left(2 \sqrt{1-2 a_{1}} B_{m} x\right), \\
S_{m}(x) & =\sin \left(2 \sqrt{1-2 a_{1}} B_{m} x\right),
\end{aligned}
$$

with $a_{1}$ being an arbitrary real number within the interval $0<a_{1}<\frac{1}{2}$. The velocity is

$$
v_{m}=\sum_{n=1}^{\infty} 4^{n} \alpha_{2 n+1} c^{2 n+1}\left(1+\sum_{r=1}^{n} \frac{(2 r-1) ! ! a_{1}^{r}}{r !}\right) .
$$

This can be written in closed form:

$$
\begin{aligned}
v_{m}= & \sum_{n=1}^{\infty} 4^{n} \alpha_{2 n+1} c^{2 n+1}\left[\frac{1}{\sqrt{1-2 a_{1}}}-a_{1}^{n+1} \frac{(2 n+1) ! !}{(n+1) !}{ }_{2} F_{1}\right. \\
& \left.\times\left(1, n+\frac{3}{2} ; n+2 ; 2 a_{1}\right)\right]
\end{aligned}
$$

Thus, we have

$$
\begin{aligned}
v_{m}= & 4 c^{3}\left(a_{1}+1\right) \alpha_{3}+8 c^{5}\left(3 a_{1}^{2}+2 a_{1}+2\right) \alpha_{5} \\
& +32 c^{7}\left(5 a_{1}^{3}+3 a_{1}^{2}+2 a_{1}+2\right) \alpha_{7} \\
& +32 c^{9}\left(35 a_{1}^{4}+20 a_{1}^{3}+12 a_{1}^{2}+8 a_{1}+8\right) \alpha_{9} \\
& +128 c^{11}\left(63 a_{1}^{5}+35 a_{1}^{4}+20 a_{1}^{3}+12 a_{1}^{2}+8 a_{1}+8\right) \alpha_{11} \\
& +\cdots .
\end{aligned}
$$

The result in Eq. (24) can also be expressed as

$$
\begin{aligned}
v_{m}= & \sum_{n=1}^{\infty} \frac{2^{n-1} \alpha_{2 n+1} c^{2 n+1}}{n ! \sqrt{1-2 a_{1}}} \\
& \left.\times\left[2^{n+1} n !-(2 n+1) ! ! \mathcal{B}_{1 / 2}\left(2 a_{1}, n+1\right)\right)\right],
\end{aligned}
$$

where the incomplete beta function $\mathcal{B}_{z}(a, b)$ is defined as $\int_{0}^{z} t^{a}(1-t)^{b-1} d t$.
For the upper point in the parameter range, i.e., for $a_{1} \rightarrow \frac{1}{2}$, we have

$$
\begin{aligned}
\lim _{a_{1} \rightarrow \frac{1}{2}} v_{m}= & \sum_{n=1}^{\infty} c^{2 n+1} \frac{(2 n+1) ! \alpha_{2 n+1}}{(n !)^{2}} \\
= & 6 c^{3} \alpha_{3}+30 c^{5} \alpha_{5}+140 c^{7} \alpha_{7} \\
& +630 c^{9} \alpha_{9}+2772 c^{11} \alpha_{11}+\cdots,
\end{aligned}
$$

again agreeing with the rogue wave result, Eq. (18).

The stretching factor $B_{m}$ is given by

$$
\begin{aligned}
B_{m}= & 2 \sum_{n=0}^{\infty} 4^{n} \alpha_{2 n+2} c^{2 n+2}\left[\frac{1}{\sqrt{1-2 a_{1}}}\right. \\
& \left.-a_{1}^{n+1} \frac{(2 n+1) ! !}{(n+1) !}{ }_{2} F_{1}\left(1, n+\frac{3}{2} ; n+2 ; 2 a_{1}\right)\right] .
\end{aligned}
$$

Further,

$$
\begin{aligned}
\lim _{a_{1} \rightarrow \frac{1}{2}} B_{m}= & 2 c^{2} \alpha_{2}+12 c^{4} \alpha_{4}+60 c^{6} \alpha_{6} \\
& +280 c^{8} \alpha_{8}+1260 c^{10} \alpha_{10}+\cdots,
\end{aligned}
$$

i.e., it is the rogue wave result of Eq. (16). The phase is

$$
\begin{aligned}
\phi_{m}= & \sum_{n=1}^{\infty} \frac{(2 n) !}{(n !)^{2}} \alpha_{2 n} c^{2 n}\left(2 a_{1}\right)^{n} \\
= & 2 a_{1}\left(2 c^{2} \alpha_{2}+12 a_{1} c^{4} \alpha_{4}+80 a_{1}^{2} c^{6} \alpha_{6}\right. \\
& \left.+560 a_{1}^{3} c^{8} \alpha_{8}+4032 a_{1}^{4} c^{10} \alpha_{10}+\cdots\right),
\end{aligned}
$$

recalling that we usually set $\alpha_{2}=\frac{1}{2}$. For the upper point in the parameter range,

$$
\begin{aligned}
\lim _{a_{1} \rightarrow \frac{1}{2}} \phi_{m}= & 2 c^{2} \alpha_{2}+6 c^{4} \alpha_{4}+20 c^{6} \alpha_{6} \\
& +70 c^{8} \alpha_{8}+252 c^{10} \alpha_{10}+\cdots,
\end{aligned}
$$

i.e., it is the rogue wave result of Eq. (17). 


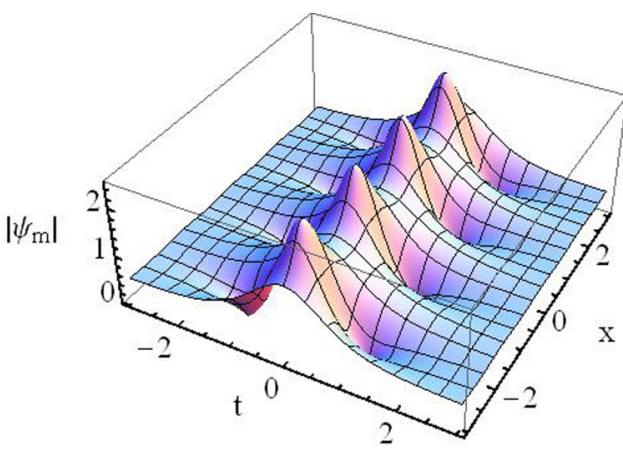

FIG. 7. Plot of the Kuznetsov-Ma breather solution given by Eq. (22). It is a solution of Eq. (7), with $a_{1}=\frac{1}{8}, c=1, \alpha_{4}=\frac{1}{4}$, and all other $\alpha_{j}$ 's being zero.

So if we only consider odd-order equations, i.e., those with coefficients $\alpha_{3}, \alpha_{5}, \alpha_{7}$, etc., then only the velocity changes, while the stretching factor $B_{m}$ and the phase $\phi_{m}$ are zero. This makes $\psi_{m}$ of Eq. (22) real. If we only consider evenorder equations, i.e., those with coefficients $\alpha_{2}, \alpha_{4}, \alpha_{6}$, etc., then only the stretching factor $B_{m}$ and the phase $\phi_{m}$ change, while the velocity remains equal to zero. Thus, the rogue wave given by Eq. (15) can also be obtained as the upper parameter $\left(a_{1} \rightarrow 1 / 2\right)$ limit of the Kuznetsov-Ma breather of Eq. (22). Again here, if we have only even-numbered equations, then $v_{m}=0$, and the breather solution resembles that of the NLSE. An example is given in Fig. 7.

If we have only odd numbered equations, then $\phi_{m}=$ $B_{m}=0$, and the solution $\psi_{m}(x, t)$ of Eq. (22) becomes real-valued. An example is given in Fig. 8. In contrast to the odd-equations' Akhmediev breathers, these contain no trigonometric functions and hence do not feature periodicity. We describe them as solutions related to the Kuznetsov-Ma breather. They resemble a moving soliton on a background, like the rogue wave shown in Fig. 2.

\section{PERIODIC SOLUTIONS}

\section{A. Elliptic $d n$ solutions}

The NLSE $d n$ solution has been given in Eq. (3.65) of Ref. [2]. We are now in a position to give periodic solutions of the full infinite equation, where we recall that $\alpha_{2}$ and all other

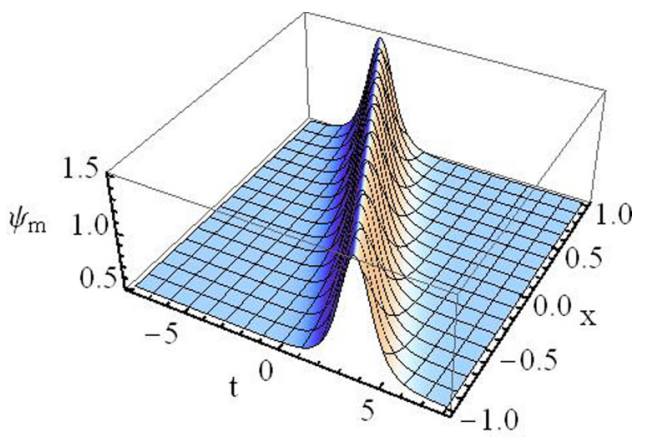

FIG. 8. Plot of the nonbreathing solution, Eq. (22). It is a solution of Eq. (7), with $a_{1}=\frac{1}{8}, c=-1, \alpha_{5}=\frac{1}{5}$, and all other $\alpha_{j}$ 's being zero. The background level is $\frac{1}{2}$ and the maximum value is $\frac{3}{2}$. coefficients $\alpha_{j}$ are arbitrary. It is given by

$$
\psi_{s}=c \exp \left(i x \phi_{d}\right) d n\left(c t+x v_{e}, m\right)
$$

where $d n$ is a Jacobi elliptic function [23], with real modulus $m$ such that $0<m<1$. For the definition of $m$, we have $d n(y, m)=1-\frac{1}{2} m y^{2}+\cdots$. The phase term is

$$
\phi_{d}=\sum_{n=1}^{\infty} \alpha_{2 n} c^{2 n} \frac{(2 n) !}{(n !)^{2}}{ }_{2} F_{1}(-n,-n ;-2 n ; m) .
$$

We find that this can be expressed in terms of $P_{n}$, the set of orthogonal Legendre polynomials of the first kind:

$$
\phi_{d}=\sum_{n=1}^{\infty} \alpha_{2 n} c^{2 n} m^{n} P_{n}\left(\frac{2}{m}-1\right) .
$$

These well-known polynomials are $P_{1}(y)=y, P_{2}(y)=\frac{1}{2}$ $\left(3 y^{2}-1\right), \quad P_{3}(y)=\frac{1}{2} y\left(5 y^{2}-3\right), \quad P_{4}(y)=$ $\frac{1}{8}\left(35 y^{4}-30 y^{2}+3\right), \quad P_{5}(y)=\frac{1}{8} y\left(63 y^{4}-70 y^{2}+15\right)$, etc. Thus,

$$
\begin{aligned}
\phi_{d}= & (2-m) c^{2} \alpha_{2}+\left(6-6 m+m^{2}\right) c^{4} \alpha_{4} \\
& +\left(20-30 m+12 m^{2}-m^{3}\right) c^{6} \alpha_{6}+(70-140 m \\
& \left.+90 m^{2}-20 m^{3}+m^{4}\right) c^{8} \alpha_{8}+(252-630 m \\
& \left.+560 m^{2}-210 m^{3}+30 m^{4}-m^{5}\right) c^{10} \alpha_{10}+\cdots
\end{aligned}
$$

Further, the velocity is

$$
v_{e}=\sum_{n=1}^{\infty} \alpha_{2 n+1} c^{2 n+1} \frac{(2 n) !}{(n !)^{2}}{ }_{2} F_{1}(-n,-n ;-2 n ; m) .
$$

Similarly, this can be simplified to

$$
v_{e}=\sum_{n=1}^{\infty} \alpha_{2 n+1} c^{2 n+1} m^{n} P_{n}\left(\frac{2}{m}-1\right),
$$

where $P_{n}$ is a member of the same set of orthogonal Legendre polynomials of the first kind. So

$$
\begin{aligned}
v_{e}= & (2-m) c^{3} \alpha_{3}+\left(6-6 m+m^{2}\right) c^{5} \alpha_{5}+(20 \\
- & \left.30 m+12 m^{2}-m^{3}\right) c^{7} \alpha_{7}+(70-140 m \\
& \left.+90 m^{2}-20 m^{3}+m^{4}\right) c^{9} \alpha_{9}+(252-630 m \\
& \left.+560 m^{2}-210 m^{3}+30 m^{4}-m^{5}\right) c^{11} \alpha_{11}+\cdots
\end{aligned}
$$

As with most solutions, the even-order equations affect the phase, while the odd-order equations affect the velocity. In this case, the solution functions have the same form, though the set of coefficients (the $\alpha_{j}$ 's) differ. If we have only odd-label equations, then $\phi_{d}=0$ and the solution of Eq. (29) is real.

If $m=1$ we have $P_{n}(1)=1$, and

$$
c e^{i \phi_{d} x} d n\left(c t+v_{e} x, 1\right)=c e^{i \phi_{s} x} \operatorname{sech}\left(t+v_{s} x\right),
$$

since $\phi_{d}(m=1)=\sum_{n=1}^{\infty} \alpha_{2 n}$, agreeing with Eq. (13), and $v_{e}=\sum_{n=1}^{\infty} \alpha_{2 n+1}$, agreeing with Eq. (14). Thus, we have reproduced the fundamental "sech" soliton result covering all operators. 


\section{B. Elliptic $c n$ solutions}

The NLSE $c n$ solution has been given by Eq. (3.66) of Ref. [2]. We now give the elliptic $c n$ solution of the full infinite equation. We can write it in a convenient way using hyperbolic functions as follows:

$$
\psi_{s}=\frac{c}{\sqrt{2}} \operatorname{coth}(\zeta) e^{i \phi_{c} x} c n\left[\frac{c t+x v_{c}}{\sinh (\zeta)}, \frac{1}{2} \cosh ^{2}(\zeta)\right]
$$

where $c n$ is a Jacobi elliptic function [23], with $\zeta$ real. With our modulus definition, $c n(y, m)=1-\frac{1}{2} y^{2}+\frac{1}{6}\left(\frac{1}{4}+m\right) y^{4}+$ $\cdots$. The phase term can be expressed in terms of $P_{n}$, the set of orthogonal Legendre polynomials of the first kind:

$$
\begin{aligned}
\phi_{c}= & \sum_{n=1}^{\infty} \alpha_{2 n} c^{2 n} \sinh ^{-2 n}(\zeta) P_{n}\left(\sinh ^{2}(\zeta)\right) \\
= & \alpha_{2} c^{2}+\frac{\alpha_{4}}{2} c^{4}\left[3 \sinh ^{4}(\zeta)-1\right] \operatorname{csch}^{4}(\zeta) \\
& +\frac{\alpha_{6}}{2} c^{6}\left[5 \sinh ^{6}(\zeta)-3 \sinh ^{2}(\zeta)\right] \operatorname{csch}^{6}(\zeta) \\
& +\frac{\alpha_{8}}{8} c^{8}\left[35 \sinh ^{8}(\zeta)-30 \sinh ^{4}(\zeta)+3\right] \operatorname{csch}^{8}(\zeta) \\
& +\frac{\alpha_{10}}{8} c^{10}\left[63 \sinh ^{10}(\zeta)-70 \sinh ^{6}(\zeta)\right. \\
& \left.+15 \sinh ^{2}(\zeta)\right] \operatorname{csch}^{10}(\zeta)+\cdots
\end{aligned}
$$

This can be reexpressed in a more compact form:

$$
\begin{aligned}
\phi_{c}= & \alpha_{2} c^{2}+\frac{\alpha_{4}}{2} c^{4}\left[3-\operatorname{csch}^{4}(\zeta)\right]+\frac{\alpha_{6}}{2} c^{6}\left[5-3 \operatorname{csch}^{4}(\zeta)\right] \\
& +\frac{\alpha_{8}}{8} c^{8}\left[35-30 \operatorname{csch}^{4}(\zeta)+3 \operatorname{csch}^{8}(\zeta)\right] \\
& +\frac{\alpha_{10}}{8} c^{10}\left[63-70 \operatorname{csch}^{4}(\zeta)+15 \operatorname{csch}^{8}(\zeta)\right]+\cdots
\end{aligned}
$$

Similarly, the velocity is

$$
\begin{aligned}
v_{c}= & \sum_{n=1}^{\infty} \alpha_{2 n+1} c^{2 n+1} \sinh ^{-2 n}(\zeta) P_{n}\left[\sinh ^{2}(\zeta)\right] \\
= & \alpha_{3} c^{3}+\frac{\alpha_{5}}{2} c^{5}\left[3-\operatorname{csch}^{4}(\zeta)\right]+\frac{\alpha_{7}}{2} c^{7}\left[5-3 \operatorname{csch}^{4}(\zeta)\right] \\
& +\frac{\alpha_{9}}{8} c^{9}\left[35-30 \operatorname{csch}^{4}(\zeta)+3 \operatorname{csch}^{8}(\zeta)\right] \\
& +\frac{\alpha_{11}}{8} c^{11}\left[63-70 \operatorname{csch}^{4}(\zeta)+15 \operatorname{csch}^{8}(\zeta)\right]+\cdots,
\end{aligned}
$$

where $P_{n}$ is a member of the same set of orthogonal Legendre polynomials.

On the other hand, the solution can be written without hyperbolic functions:

$$
\psi_{s}=\frac{c}{\sqrt{2}} \sqrt{s+1} e^{i \phi_{c} x} c n\left[\sqrt{s}\left(c t+x v_{c}\right), \frac{1}{2}\left(1+s^{-1}\right)\right] .
$$

Then

$$
\begin{aligned}
\phi_{c} & =\sum_{n=1}^{\infty} \alpha_{2 n} c^{2 n} s^{n} P_{n}\left(\frac{1}{s}\right) \\
& =c^{2} \alpha_{2}+\frac{\alpha_{4}}{2} c^{4}\left(3-s^{2}\right)+\frac{\alpha_{6}}{2} c^{6}\left(5-3 s^{2}\right)
\end{aligned}
$$

$$
\begin{aligned}
& +\frac{\alpha_{8}}{8} c^{8}\left(35-30 s^{2}+3 s^{4}\right) \\
& +\frac{\alpha_{10}}{8} c^{10}\left(63-70 s^{2}+15 s^{4}\right)+\cdots
\end{aligned}
$$

and

$$
\begin{aligned}
v_{c}= & \sum_{n=1}^{\infty} \alpha_{2 n+1} c^{2 n+1} s^{n} P_{n}\left(\frac{1}{s}\right) \\
= & c^{3} \alpha_{3}+\frac{\alpha_{5}}{2} c^{5}\left(3-s^{2}\right)+\frac{\alpha_{7}}{2} c^{7}\left(5-3 s^{2}\right) \\
& +\frac{\alpha_{9}}{8} c^{9}\left(35-30 s^{2}+3 s^{4}\right) \\
& +\frac{\alpha_{11}}{8} c^{11}\left(63-70 s^{2}+15 s^{4}\right)+\cdots
\end{aligned}
$$

Again, the even-order equations affect the phase, while the odd-order equations affect the velocity. If we have only odd-label equations, then $\phi_{c}=0$ and solution of Eq. (34) is real. In this case, the solutions have the same form, with the set of coefficients (the $\alpha_{j}$ 's) being different. If $s=1$, i.e., $\sinh (\zeta)=1$, we have $P_{n}(1)=1$, so the solution given in Eq. (37) reduces to Eq. (12), viz., $c e^{i \phi_{s} x} \operatorname{sech}\left(t+v_{s} x\right)$, as is the case in Sec. VI A.

\section{THE CASE OF $x$-DEPENDENT COEFFICIENTS}

\section{A. Solitons}

We have considered the coefficients to be constants, but we now allow them to vary on propagation, so that $\alpha_{m}=$ $\alpha_{m}(x)$. In a fiber, this would correspond to different sections possessing different physical and optical characteristics. For example, suppose that just one of the coefficients, viz., $\alpha_{2 j}(x)$, in Eq. (6) is nonzero. Then

$$
i \psi_{x}+\alpha_{2 j}(x) K_{2 j}(x, t)=0,
$$

for a particular $j$. We note that $K_{2 j}(x, t)$ contains no derivatives with respect to $x$. We now transform to a new variable, $X$, such that

$$
\frac{d X}{d x}=\alpha_{2 j}(x) \text {, i.e., } X=\int \alpha_{2 j}(x) d x .
$$

Then $i \psi_{X}+K_{2 j}(X, t)=0$. Here the coefficient is a constant, viz., unity, and we can use the constant-coefficient solutions already found, simply by making the following replacement: $\alpha_{2 j} x \rightarrow \int \alpha_{2 j}(x) d x$. The velocities, stretching factors, and phases are modified in this way for all the solutions given above. For example, if we take $j=1$, we have the NLS only, $K_{2}=\psi_{t t}+2|\psi|^{2} \psi$. The soliton solution is, from Eq. (12), $\psi=\exp \left[i \alpha_{2} x\right] \operatorname{sech}(t)$. When $\alpha_{2}=\alpha_{2}(x)$, we have $\psi=\exp \left[i \int \alpha_{2}(x) d x\right] \operatorname{sech}(t)$.

We can generalize this by allowing all operator coefficients to be nonzero and to be functions of $x$. Then the soliton solution 


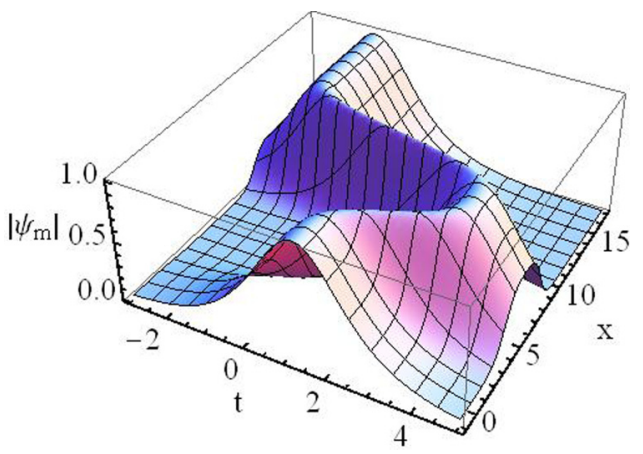

FIG. 9. Plot of the soliton, Eq. (40), moving under the influence of operators with variable coefficients.

of maximum amplitude $c$ is

$$
\psi_{m}=c \exp \left[i \sum_{n=1}^{\infty} c^{2 n} J_{2 n}(x)\right] \operatorname{sech}\left[t+\sum_{n=1}^{\infty} c^{2 n+1} J_{2 n+1}(x)\right],
$$

where $J_{m}(x)=\int \alpha_{m}(x) d x$. If each coefficient is constant, then $J_{m}(x)=\alpha_{m} x$, and

$$
\psi_{m}=c \exp \left[i x \sum_{n=1}^{\infty} c^{2 n} \alpha_{2 n}\right] \operatorname{sech}\left[t+x \sum_{n=1}^{\infty} c^{2 n+1} \alpha_{2 n+1}\right],
$$

as in Eqs. (13) and (14).

To plot an example, let us use Gaussian functions to switch the operators "on" and "off" during soliton propagation. We set $\alpha_{2}(x)=\exp \left[-\frac{1}{2} x^{2}\right], \alpha_{3}(x)=-\exp \left[-\frac{1}{2}(x-3)^{2}\right], \alpha_{4}(x)=$ $\exp \left[\frac{1}{2}(x-6)^{2}\right]$, and $\alpha_{5}(x)=2 \exp \left[-\frac{1}{2}(x-9)^{2}\right]$. Hence $J_{2}=$ $\sqrt{\frac{\pi}{2}} \operatorname{erf}\left(\frac{x}{\sqrt{2}}\right)$, etc. We plot the solution, from Eq. (40), in Fig. 9. Clearly, the third-order operator, mediated by $\alpha_{3}$, moves the soliton towards the right, while the fifth-order operator, mediated by $\alpha_{5}$, moves the soliton towards the left. The other two operators affect phase only, and not velocity. In parts where the Gaussians are almost zero, the soliton propagates with unchanged velocity and phase.

Using Eq. (15), or varying $\alpha_{2}(x)$, the NLS unit-background rogue wave becomes

$$
\psi(x, t)=\left[4 \frac{1+4 i J_{2}(x)}{1+4 t^{2}+16 J_{2}^{2}(x)}-1\right] e^{2 i J_{2}(x)} .
$$

\section{B. Kuznetsov-Ma breathers}

We now consider a Kuznetsov-Ma breather, but allow for variable coefficients. We take

$$
\begin{aligned}
& \alpha_{2}(x)=\gamma \operatorname{sech}^{2}\left(\frac{x-10}{8}\right), \alpha_{3}(x)=-\frac{\gamma}{4} \operatorname{sech}^{2}\left(\frac{x-50}{8}\right), \\
& \alpha_{4}(x)=\frac{\gamma}{6} \operatorname{sech}^{2}\left(\frac{x-90}{8}\right) .
\end{aligned}
$$

We can still use the result of Eq. (22), again with each $\alpha_{n} x$ with $\int \alpha_{n}(x) d x$. Here we take $a_{1}=1 / 8$. Thus $v_{m}$ of Eq. (24) is replaced by $v_{m}=-9 \gamma \tanh \left(\frac{x-50}{8}\right), B_{m}$ of Eq. (27) is replaced by $B_{m}=4 \gamma\left[4 \tanh \left(\frac{x-10}{8}\right)+3 \tanh \left(\frac{x-90}{8}\right)\right]$, and

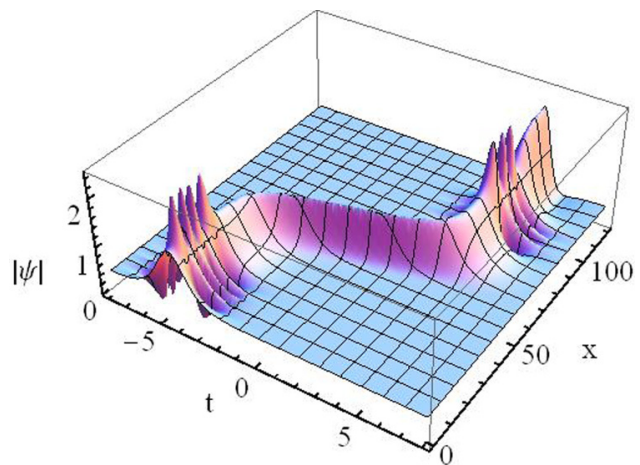

FIG. 10. Plot of the Kuznetsov-Ma breather in the case of variable coefficients. The pattern shows the influence of three operators with coefficients which vary on propagation.

$\phi_{m}$ of Eq. (28) is replaced by

$$
\phi_{m}=4 \gamma \tanh \left(\frac{x-10}{8}\right)+\frac{\gamma}{2} \tanh \left(\frac{x-90}{8}\right) .
$$

This example, with $\gamma=\frac{1}{2}$, is shown in Fig. 10. In this figure, the angled propagation (at around $x=50$ ) is due to nonzero velocity $\left(v_{m}\right)$ being introduced by the third-order operator, with coefficient $\alpha_{3}(x)$, as this operator differs strongly from zero only around $x=50$. The breather peaks, around $x=10$ and $x=90$, are due to the influence of coefficients $\alpha_{2}(x)$ and $\alpha_{4}(x)$, respectively, as these coefficients differ substantially from zero only near these values of $x$. Hence, as in Fig. 7, peaks occur in these regions.

\section{CONCLUSION}

In conclusion, we have presented the infinite integrable NLSE hierarchy beyond the LPD equation, which is a particular fourth-order case of the hierarchy. Specifically, we have presented explicit forms of the equations and given generalized soliton solutions, plane wave solutions, Akhmediev breathers, Kuznetsov-Ma breathers, and periodic and rogue wave solutions for this infinite-order hierarchy. We have found that even-order equations in the set affect phase and stretching factors in the solutions, while odd-order equations affect the velocities. Hence odd-order equation solutions can be real functions, while even-order equation solutions are always complex. Of special interest is the possibility of using variable coefficients in the hierarchy to influence evolution dynamics. Examples of such evolution are given.

\section{ACKNOWLEDGMENTS}

The authors acknowledge the support of the Australian Research Council (Discovery Project No. DP140100265). N.A. and A.A. acknowledge support from the Volkswagen Stiftung; A.C. acknowledges support from an Endeavour Postgraduate Award. U.B. gratefully acknowledges support by the Einstein Center for Mathematics Berlin under Project No. D-OT2. 


\section{APPENDIX}

Following Eq. (10), we now present $K_{7}$, the seventh-order $(j=7)$, i.e., heptic, operator (starting with the seventh-order derivative):

$$
\begin{aligned}
K_{7}[\psi]= & \psi_{t t t t t t t}+70 \psi_{t t}^{2} \psi_{t}^{*}+112 \psi_{t}\left|\psi_{t t}\right|^{2}+98\left|\psi_{t}\right|^{2} \psi_{t t t}+70 \psi^{2}\left[\psi_{t}\left[\left(\psi_{t}^{*}\right)^{2}+2 \psi^{*} \psi_{t t}^{*}\right]\right. \\
& \left.+\psi^{*}\left(2 \psi_{t t} \psi_{t}^{*}+\psi_{t t t} \psi^{*}\right)\right]+28 \psi_{t}^{2} \psi_{t t t}^{*}+14 \psi\left[\psi^{*}\left(20\left|\psi_{t}\right|^{2} \psi_{t}+\psi_{t t t t t}\right)+3 \psi_{t t t} \psi_{t t}^{*}\right. \\
& \left.+2 \psi_{t t} \psi_{t t t}^{*}+2 \psi_{t t t t} \psi_{t}^{*}+\psi_{t} \psi_{t t t t}^{*}+20 \psi_{t} \psi_{t t}\left(\psi^{*}\right)^{2}\right]+140|\psi|^{6} \psi_{t}+70 \psi_{t}^{3}\left(\psi^{*}\right)^{2}+14\left(5 \psi_{t t} \psi_{t t t}+3 \psi_{t} \psi_{t t t t}\right) \psi^{*} .
\end{aligned}
$$

There is an infinite number of higher-order operators. The highest one that we provide here is $K_{8}$, which is the eighth-order $(j=8)$, i.e., octic, operator (starting with the eighth-order derivative):

$$
\begin{aligned}
K_{8}[\psi]= & \psi_{t t t t t t t}+14 \psi^{3}\left[40\left|\psi_{t}\right|^{2}\left(\psi^{*}\right)^{2}+20 \psi_{t t}\left(\psi^{*}\right)^{3}+2 \psi_{t t t t}^{*} \psi^{*}+3\left(\psi_{t t}^{*}\right)^{2}+4 \psi_{t}^{*} \psi_{t t t}^{*}\right] \\
& +\psi^{2}\left[28 \psi^{*}\left(14 \psi_{t t} \psi_{t t}^{*}+11 \psi_{t t t} \psi_{t}^{*}+6 \psi_{t} \psi_{t t t}^{*}\right)+238 \psi_{t t}\left(\psi_{t}^{*}\right)^{2}+336\left|\psi_{t}\right|^{2} \psi_{t t}^{*}+560 \psi_{t}^{2}\left(\psi^{*}\right)^{3}\right. \\
& \left.+98 \psi_{t t t t}\left(\psi^{*}\right)^{2}+2 \psi_{t t t t t t}^{*}\right]+2 \psi\left\{21 \psi_{t}^{2}\left[9\left(\psi_{t}^{*}\right)^{2}+14 \psi^{*} \psi_{t t}^{*}\right]+\psi_{t}\left[728 \psi_{t t} \psi_{t}^{*} \psi^{*}+238 \psi_{t t t}\left(\psi^{*}\right)^{2}\right.\right. \\
& \left.\left.+6 \psi_{t t t t t}^{*}\right]+34\left|\psi_{t t t}\right|^{2}+36 \psi_{t t t t} \psi_{t t}^{*}+22 \psi_{t t} \psi_{t t t t}^{*}+20 \psi_{t t t t t} \psi_{t}^{*}+161 \psi_{t t}^{2}\left(\psi^{*}\right)^{2}+8 \psi_{t t t t t} \psi^{*}\right\} \\
& +182 \psi_{t t}\left|\psi_{t t}\right|^{2}+308 \psi_{t t} \psi_{t t t} \psi_{t}^{*}+252 \psi_{t} \psi_{t t t} \psi_{t t}^{*}+196 \psi_{t} \psi_{t t} \psi_{t t t}^{*}+168 \psi_{t} \psi_{t t t t} \psi_{t}^{*}+42 \psi_{t}^{2} \psi_{t t t t}^{*} \\
& +14 \psi^{*}\left(30 \psi_{t}^{3} \psi_{t}^{*}+4 \psi_{t t t t t} \psi_{t}+5 \psi_{t t t}^{2}+8 \psi_{t t} \psi_{t t t t}\right)+490 \psi_{t}^{2} \psi_{t t}\left(\psi^{*}\right)^{2}+140 \psi^{4} \psi^{*}\left[\left(\psi_{t}^{*}\right)^{2}+\psi^{*} \psi_{t t}^{*}\right]+70 \psi|\psi|^{8} .
\end{aligned}
$$

[1] V. E. Zakharov and A. B. Shabat, J. Exp. Theor. Phys. 34, 62 (1972).

[2] N. Akhmediev and A. Ankiewicz, Solitons, Nonlinear Pulses and Beams, (Chapman \& Hall, London, 1997).

[3] R. Sahadevan and L. Nalinidevi, J. Nonlinear Math. Phys. 17, 379 (2010).

[4] T. Kano, J. Phys. Soc. Jpn. 58, 4322 (1989).

[5] Luc Bergé, Phys. Rep. 303, 259 (1998).

[6] A. Ankiewicz, J. M. Soto-Crespo, and N. Akhmediev, Phys. Rev. E 81, 046602 (2010).

[7] A. Ankiewicz and N. Akhmediev, Phys. Lett. A 378, 358 (2014).

[8] A. Ankiewicz, Yan Wang, S. Wabnitz, and N. Akhmediev, Phys. Rev. E 89, 012907 (2014).

[9] A. Ankiewicz, D. J. Kedziora, and N. Akhmediev, Phys. Lett. A 375, 2782 (2011).

[10] S. M. Hoseini and T. R. Marchant, Wave Motion 44, 92 (2006).

[11] A. Hasegawa and F. Tappert, Appl. Phys. Lett. 23, 142 (1973).

[12] L. F. Mollenauer, R. H. Stolen, and J. P. Gordon, Phys. Rev. Lett. 45, 1095 (1980).

[13] G. P. Agrawal, Nonlinear Fiber Optics, 4th ed., Optics and Photonics series (Academic, San Diego, 2006), Sec. 5.5.3.
[14] V. B. Matveev and M. Salle, Darboux Transformations and Solitons (Springer-Verlag, Berlin, Heidelberg, 1991)

[15] R. Hirota, J. Math. Phys. 14, 805 (1973).

[16] M. Lakshmanan, K. Porsezian, and M. Daniel, Phys. Lett. A 133, 483 (1988).

[17] K. Porsezian, M. Daniel, and M. Lakshmanan, J. Math. Phys. 33, 1807 (1992).

[18] K. Porsezian, Phys. Rev. E 55, 3785 (1997).

[19] P. Wang, B. Tian, W.-J. Liu, Q.-X. Qu, M. Li, and K. Sun, Eur. Phys. J. D 61, 701 (2011).

[20] R. Sahadevan and L. Nalinidevi, J. Math. Phys. 50, 053505 (2009).

[21] A. Ankiewicz, J. Nonlinear Opt. Phys. Mater. 4, 857 (1995).

[22] D. H. Peregrine, J. Aust. Math. Soc., Ser. B 25, 16 (1983).

[23] M. Abramowitz and I. A. Stegun (Editors), Handbook of Mathematical Functions with Formulas, Graphs, and Mathematical Tables (Dover, New York, 1972), pp. 555-566.

[24] L. H. Wang, K. Porsezian, and J. S. He, Phys. Rev. E 87, 053202 (2013). 